\section{PO 8559 ASSESSMENT OF THE EFFECT OF RVSV REPLICATION ON NON-EBOLA CIRCULATING ANTIBODIES IN RVSV-ZEBOV-GP-VACCINATED INDIVIDUALS IN LAMBARÉNÉ, GABON}

${ }^{1,3}$ Armel V Ndong Mbouna, ${ }^{2,3}$ Selidji T Agnandji. ${ }^{1}$ Centre de Recherche de Lambarene, Gabon; ${ }^{2}$ Institut für Tropenmedizin, Universitätsklinikum Tübingen, Germany; ${ }^{3}$ Centre de Recherches Médicales de Lambaréné (CERMEL), Lambaréné, Gabon

\subsection{6/bmjgh-2019-EDC.138}

Background The West Africa Ebola virus disease (EVD) outbreak between 2015 and 2016 accelerated the need for safe and effective vaccines. Among candidate vaccines in clinical development, the recombinant Vesicular stomatitis virus (VSV) vectored with the Ebola virus (EBOV) glycoprotein (rVSVZEBOV-GP) vaccine showed acceptable safety and promising immunogenicity results across diverse settings.

Baseline screening data from the phase I trial of this vaccine in Lambaréné, Gabon, established that prior to vaccination about 21\% (33/155) and $8 \%(12 / 155)$ of adults had naturally acquired antibodies to infectious ZEBOV particle and ZEBOV-GP, respectively. In participants with prior ZEBOV(GP) antibodies, post-vaccination antibodies titres were significantly higher 56 days following vaccination with doses of $3 \times 10^{3}, 3 \times 10^{4}$, and $3 \times 10^{6}$ PFU compared to those without.

Our study seeks to investigate rVSV vector non-specific boosting of naturally acquired antibodies to other viral infections (dengue virus $1-4$, and yellow fever virus).

Methods We measured antibodies titres to Dengue (serotypes 14) and yellow fever infection at baseline, 28 and 56 days after injection in a total of 155 serum samples from vaccinees receiving various doses of rVSV-ZEBOV-GP using ELISA technique.

Results Preliminary results were presented at the meeting. Conclusion Our results confirm rVSV vector non-specific replication on non ZEBOV-GP circulating antibodies in Lambaréné vaccinees and potential boosting action on naturally acquired dengue virus (serotypes 1-4) and yellow fever virus antibodies.

\section{PO 8562 SECRETED OOKINETE PROTEIN AS A MARKER OF INFECTIOUS BITES FOR ASSESSING TRANSMISSION DYNAMICS AT THE POPULATION LEVEL}

Kingsley Badu. Kwame Nkrumah University of Science and Technology, Kumasi, Ghana

\subsection{6/bmjgh-2019-EDC.139}

Background As malaria transmission intensity declines, the heterogeneity in infectious burden becomes pronounced. There is thus the need for more sensitive tools to identify micro-geographic areas of higher risk for targeted interventions. We sought to evaluate several immunogenic peptides of $P$. falciparum, secreted ookinete and sporozoite proteins (PSOP24) and possibly validate specific short sequence immunogenic peptides as an infectious bite marker for assessing malaria transmission intensity and dynamics. Methods We conducted four cross-sectional serological and parasitological surveys within one peri-urban and one rural community about $3 \mathrm{~km}$ apart, in South-western Ghana. The field surveys were conducted from November 2012 to July 2014 across dry and rainy seasons. Several bioinformatics models were used to predict the immunogenic epitopes of PSOP24 peptides. Total IgG antibody response were determined for three most promising peptides (PSOP24-374, PSOP24-375 and PSOP24-377), together with MSP1 19 , CSP and salivary gland antigen. Alongside we determined parasite prevalence and density as well as the entomological inoculation rates.

Results Peptide PSOP24-377 showed seasonal variation with a twofold increase in IgG response in the high-transmission rainy season. This collaborates with the twofold increase in IgG response to the mosquito salivary antigen gSG6-P1. Also, PSOP24-377 was able to show a subtle difference from Ayeigbekorpe to Odumase during the dry season and a high seroprevalence between the two communities during the rainy season. This was in contrast with gSG6-P1 because, while PSOP24-377 measures sero-response to infectious bites, gSG6P1 measure responses to only vector exposure. The immune response variation determined by PSOP24-377 correlated with parasite prevalence and the entomological inoculation rates.

Conclusion The preliminary data points to the potential of PSOP24-377 as an infectious bite marker. This may be exploited as a routine surveillance tool for monitoring malaria transmission at the community level.

\section{PO 8563 UNCOMMONLY HIGH AND SYMPTOMATIC PLASMODIUM FALCIPARUM INFECTIONS IN GABONESE ADULTS}

${ }^{1,2,3}$ Rella Zoleko Manego*, 1,2 Eric Koehne, 1,2,3 Ghyslain Mombo-Ngoma. ${ }^{1}$ Centre de Recherches Médicales de Lambaréné (CERMEL), Gabon; 'Institute of Tropical Medicine, University of Tübingen and German Centre for Infectious Diseases (DZIF), Tübingen, Germany; ${ }^{3}$ Bernhard Nocht Institute for Tropical Medicine, World Health Organization Collaborating Centre for Arbovirus and Hemorrhagic Fever Reference and Research, Hamburg, Germany

\subsection{6/bmjgh-2019-EDC.140}

Background Malaria remains a public health issue particularly in sub-Saharan Africa with special features of seriousness in young children and pregnant women. Older children and adults reported to have acquired a semi-immune status and therefore show low parasitaemia and less pronounced symptoms if any. We aim to explore parasitaemia and symptoms in adolescents and adults with malaria compared to younger children in the Lambaréné area.

Methods We conducted a cross-sectional study in CERMEL for a month in 2018. Adults and children were screened for malaria at health facilities in Lambaréné and Fougamou and their respective surrounding villages in the central region of Gabon. Malaria infection was determined either by rapid diagnostic test (RDT) or by thick blood smear (TBS). Body temperature, history of fever, age, sex, haemoglobin level, and location were collected.

Results From 09 January to 09 February 2018, a total of 596 patients were screened and 280 (47\%) belonged to the adult population. Plasmodium falciparum infection in adults was diagnosed based on the detection of $P$. falciparum in thick blood films. Prevalence among the adults was 56\% $(n=141)$; $34 \%$ of adult patients had a parasite count between 1015$116,079 \mathrm{pf} / \mu \mathrm{l}$. Fever was found in $31 \%$ of adults and several symptoms were reported by the same patient. Vomiting, loss of appetite, and fatigue were significantly more present in the adult population than in children.

Conclusion This study shows a high rate of Plasmodium falciparum infection in adults associated to clinical symptoms and high level of parasitaemia. Adults seem to become an at-risk population, suggesting that malaria programmes should consider including adults in the implementation of a malaria prevention programme. 